\title{
New Space-Charge Methods in Accsim and Their Application to Injection in the CERN PS Booster
}

\author{
F.W. Jones, TRIUMF, 4004 Wesbrook Mall, Vancouver, V6T 2A3, Canada \\ H.O. Schönauer, CERN, CH-1211 Geneva 23, Switzerland
}

\begin{abstract}
The tracking and simulation code Accsim has recently been upgraded with new treatments of transverse space charge, one based on a conventional multiple-Fourier-transform technique and another using a new hybrid-fast-multipole (HFM) method. We present the application of the code to the study of multiturn injection in the CERN PS Booster in its LHC-injector-chain operation scenario. In particular, the ability of the HFM method to accurately model the turnby-turn stacking and subsequent development of the beam is evaluated, especially its development near the sharp cutoff produced by beam loss on the injection septum. Results are compared with measured injection efficiencies and betatron amplitude distributions.
\end{abstract}

\section{INTRODUCTION}

As part of a TRIUMF/CERN collaboration agreement, the tracking and simulation code Accsim is being developed to support the study of injection and collimation in the CERN PS Booster, in its role as part of the LHC injector chain. The code contains many useful simulation features for injection and collimation scenarios, including phase-space painting, programmed orbit bumps, rf harmonics, barrier buckets, and Monte Carlo treatments of particle interactions with stripping foils and collimator materials. These features are built on top of a 3D (6 phase-space variables) symplectic tracking engine.

Longitudinal space charge effects are included in the conventional manner by binning, smoothing, and differentation of the line density. Until recently, a complete model of transverse space-charge effects was lacking, but early-on a package a package "DQ" was added, which utilitizes amplitude binning and averaging over betatron phases to derive the effective beam potential, and thence a "fast parameterization" of the distribution of tune-shifts in the beam.

With improvements in computing resources, the desire has arisen to develop a more general, self-consistent treatment of transverse space charge, while keeping in mind the mandate of the code to run on conventional desktop computers rather than supercomputers. The present paper describes the upgrading of the code to achieve this, via new routines using field-solve/kick methods which are integrated with Accsim's matrix/thin-lens tracking. This will be followed by initial test results and some simulation results for the PS Booster.

\section{TRANSVERSE SPACE-CHARGE MODEL}

To satisfy the computing-time constraints, a $2 \frac{1}{2} \mathrm{D}$ transverse space-charge model was chosen for Accsim, where the term " $2 \frac{1}{2} \mathrm{D}$ " refers to mixed $2 \mathrm{D}$ and $3 \mathrm{D}$ aspects: the model is $2 \mathrm{D}$ in the sense that the nominal transverse spacecharge field is evaluated by viewing all macroparticles as 2-dimensional (line) charges; whereas the model is 3D in the sense that the space-charge force on a given macroparticle is scaled according to the longitudinal charge density at its position in the bunch, thus coupling the longitudinal motion into the transverse tune space.

We consider that the bunch length is much larger than the transverse beam size, and that there is no significant correlation between transverse and longitudinal distributions. In typical Accsim applications, this holds because any such correlations in the injected linac beam will be masked in the ring by the multiturn injection and phase-space painting process. Under these circumstances, the projected 2D distribution of transverse macroparticle coordinates can be condsidered representative of the local transverse beam distribution at a given location in the ring. In fact, Accsim's transfer-matrix/kick formalism already lends itself this model because it tracks in distance steps rather than time steps: each macroparticle carries a time-difference ( $\mathrm{rf}$ phase) coordinate locating it in the bunch, and the stored transverse coordinates are a "snapshot" in space rather than in time.

As in other codes, tracking with space-charge is done a successive "field-solve/particle-push" integration, where the "push" operation consists of applying angular kicks representing the force integral over the integration step, followed by matrix/thin-lens transport over the step.

\section{FIELD SOLUTION METHODS}

The rapid and accurate evaluation of the 2D space-charge field is the key to usability of the model in Accsim, and here two methods have been implemented, both founded on the usual Particle-In-Cell (PIC) scheme where the field is solved on a set of discrete mesh points, using bilinear weighting to assign macroparticle charges to the mesh and to derive the resultant forces on the macroparticles. The first field-solution method uses the Multiple-FourierTransform (MFT) technique, via routines contributed by Accsim users at BNL and ORNL[5]. The second fieldsolution method uses a new Hybrid Fast-Multipole (HFM) technique that was developed for Accsim in order to ad- 
dress beam-distribution and halo issues that may arise in short-term or long-term injection simulations.

The HFM technique utilizes the DAPIP2 package of routines developed by L. Greengard[1]. They are a robust 2D implementation of his Fast-Multipole Method (FMM) field solver, which is designed to solve the field for an arbitrary collection of discrete charges. The FMM method does not use a grid, but rather subdivides the solution domain into a heirarchical tree of square or cubic regions, in which multipole expansions of the field are computed. The regions and the multipole orders are chosen to satisfy the desired field accuracy while minimizing the computing time. This method is nominally slower than FFT-based methods, but it is superior in dealing with heterogeneous mixtures of charges and distance scales.

From FMM, it is a simple step to Accsim's HFM method: namely choosing a PIC grid on the solution domain, assigning charges to it, and giving the PIC grid charges to the FMM routine to solve, rather than giving it the set of discrete macroparticle charges. What does this achieve? Since there are usually much fewer PIC grid points that actual macroparticles, this obviously speeds up FMM so that it can compete with an FFT-based solution. The use of a PIC grid also achieves an intrinsic smoothing of the charge distribution, which greatly eases the noise problem found in FMM and direct-sum field solutions, usually dealt with by incorporating a smoothing parameter in distance reckonings.

The MFT and HFM techniques both do the same job, but there are some important differences:

- MFT must solve the field at all PIC grid points, even though many of them may have no nearby macroparticles. HFM solves the field only at the grid points that have charges on them.

- In MFT the PIC grid must be made somewhat larger than the charge ensemble, in order to provide a "guard band" against the (non-physical) implicit periodic boundary conditions. When a beam halo develops, and the PIC grid is augmented to accommodate it, there is a large uplift in computation time. HFM does not have this penalty and therefore becomes faster than MFT, at the same spatial resolution, when a significant halo is present.

- MFT normally uses a regular grid, whereas in HFM there are no restrictions on the grid: it can be irregular in spacing and of arbitrary size and shape. Where needed, additional grid points can be added to resolve fine details in the charge distribution. Particles that grow to large amplitudes and fall outside the grid are no problem: they are simply added as discrete charges to the list of charges presented to the FMM solver.

The charge-assignment and force-interpolation processes are identical for MFT and HFM, although some additional bookkeeping is required for HFM to handle possible mixtures of gridded and single-particle charges. The computational details and some comparison tests can be found in Reference [2].

\section{CODE AND LATTICE VALIDATION}

The CERN PS Booster is a period 16 triplet lattice of circumference 157.08 meters. In order to best resolve the quadrupole spacing in the triplet structure, a nominal space-charge integration step size of $0.70125 \mathrm{~m}$, or 14 steps per period, was chosen. Since the code had formerly only been tested with regular FODO lattices, where the step subdivision is much simpler, some validation runs were performed on the PSB lattice subdivision by tracking a slightly mismatched K-V beam of 10000 macroparticles (representing $\sim 10^{13}$ protons and yielding tune shifts of $\sim 0.2$ ) for 100 turns. The nominal single-particle tune, and envelope eigenfrequencies were measured by FFT's on the tracking data, as shown in Table 1.

Table 1: Comparison of predicted and measured singleparticle tunes and envelope eigenfrequencies

\begin{tabular}{|l|l|l|l|l|}
\hline Frequency & $Q_{x}$ & $Q_{y}$ & $Q_{\text {env }}^{-}$ & $Q_{\text {env }}^{+}$ \\
\hline \hline Analytical result & 4.077 & 5.371 & 8.323 & 10.920 \\
Accsim FFT & 4.09 & 5.38 & 8.32 & 10.87 \\
\hline
\end{tabular}

The results are in excellent accord with analytical predictions of the single-particle tunes by the generalized Laslett formula

$$
\Delta Q_{x, y}=\frac{r_{p} N_{I}\left(q^{2} / A\right) F_{x, y} G_{x, y} \bar{H}_{x, y}}{\pi \varepsilon_{x, y} \beta^{2} \gamma^{3} B_{f}}
$$

and of the envelope eigenfrequencies[3]

$$
\begin{aligned}
Q_{\mathrm{env}}^{2}= & 2 Q_{0 x}^{2}+2 Q_{0 y}^{2}-5 Q_{0 x} \Delta Q_{x} \\
& \pm \sqrt{\left(2 Q_{0 x}^{2}-2 Q_{0 y}^{2}\right)^{2}+\left(Q_{0 x} \Delta Q_{x}\right)^{2}},
\end{aligned}
$$

where $Q_{0 x}$ and $Q_{0 y}$ are the bare tunes (4.28 and 5.55). We also observed good tune uniformity over the K-V distribution, with $99 \%$ tune spreads of $\sim 0.01$ in each plane.

\section{CERN PS BOOSTER SIMULATION PARAMETERS}

The CERN PS Booster is one of the few proton machines using classical multiturn injection. A falling sawtoothshaped orbit bump of $49 \mathrm{~mm}$ initial horizontal amplitude and $50 \mu$ s fall time approaches a magnetic septum at 45 $\mathrm{mm}$. The linac beam is injected at $53.7 \mathrm{~mm}$ and mismatched such that its ellipse in phase space osculates the ellipse of the final circulating beam. By varying the length of the linac pulse and time-shifting the bump with respect to it, emittance and intensity can be controlled-within limits. At present the linac current is about $160 \mathrm{~mA}$ or $2.2 \times 10^{12}$ protons/turn, at an emittance of $16 \pi \mathrm{mm} \mathrm{mrad}$. There are two particularly interesting injection schemes: 
Table 2: Results of Accsim and Measurements after Injection at $50 \mathrm{MeV}$ with BeamScope.

\begin{tabular}{|l|c|c|c|}
\hline & Accsim & BeamScope & Unit \\
\hline \hline Injected beam & $1.77 \times 10^{12}$ & $1.07 \times 10^{12}$ & Protons \\
$\epsilon_{h}(95 \%)$ & 40.0 & 37.5 & $\pi \mathrm{mm} \mathrm{mr}$ \\
$\epsilon_{v}(95 \%)$ & 36.0 & 44.8 & $\pi \mathrm{mm} \mathrm{mr}$ \\
$\epsilon_{h}$ proj. $(2 \sigma)$ & 21.1 & 23.5 & $\pi \mathrm{mm} \mathrm{mr}$ \\
$\epsilon_{v}$ proj. $(2 \sigma)$ & 18.5 & 19.4 & $\pi \mathrm{mm} \mathrm{mr}$ \\
\hline
\end{tabular}

One for highest intensity (typically 13 turns injected, filling horizontal emittances of up to $300 \pi \mathrm{mm} \mathrm{mrad}$ ), and a low intensity, high brilliance beam for future LHC filling ( 3 turns and about $30 \pi \mathrm{mm}$ mrad). In this paper we concentrate on the high brilliance beam, mainly because there are less unknown factors like stopbands to be simulated, and because the shorter tracking duration allows comparison with the Agile code [4] featuring direct interaction between macroparticles.

\section{SIMULATION RESULTS}

The amplitude profiles after removal of the circulating beam from the septum are compared with profiles measured with BeamScope, the PS Booster emittance measurement device. Characteristic beam parameters are compared in Table 2 and example profiles are shown in Figure 1.
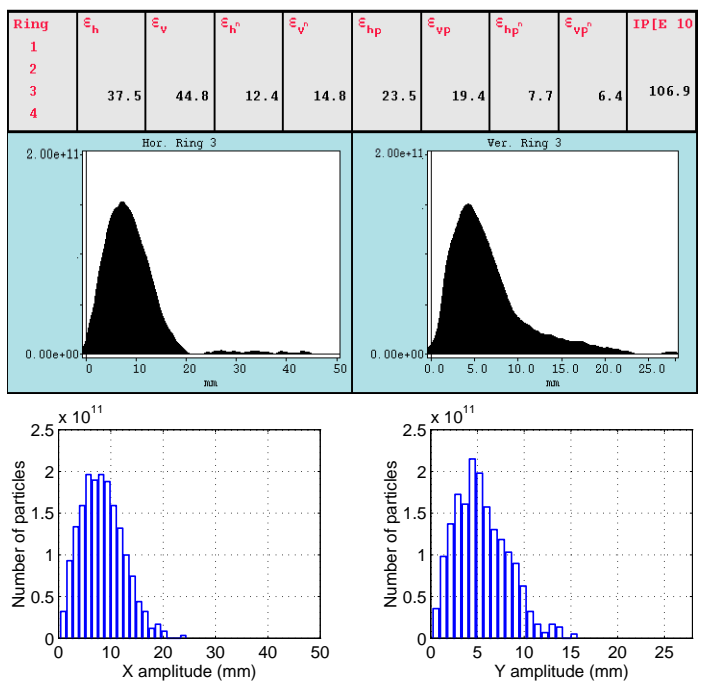

Figure 1: Comparison of BeamScope (top) and Accsim profiles

Another, already mentioned test consists in comparing phase space distributions after a few turns between Accsim and the Agile interactive lattice design code, featuring also tracking of macroparticle distributions with direct electrostatic interaction. Based on a PC under Windows95, these computations take time and are thus limited to fewturn tracking. The phase-space plots are shown in Figure 2. Real space plots, less instructive, are almost identical.
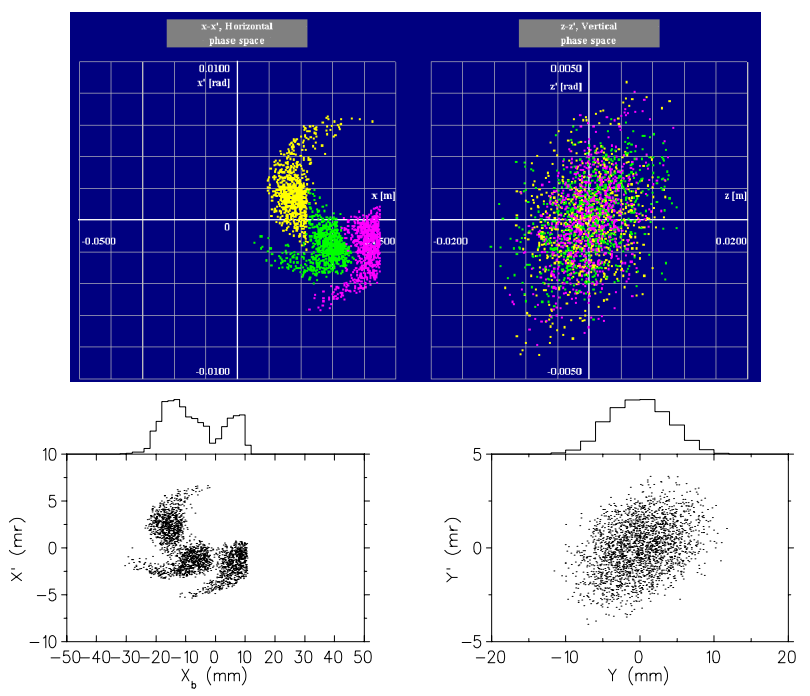

Figure 2: Comparison of Agile (top) and Accsim phasespace plots (the closed orbit deviation is removed in the Accsim plot).

\section{DISCUSSION OF RESULTS}

The BeamScope profiles show a halo in the vertical plane, which is not present in the simulation. It should be noted that BeamScope measurements take some time and cannot be performed immediately, which means that the circulating beam is subject to the machine nonlinearities during 2-3 ms. The halo is probably due to growth on one of or both the stopbands $4 Q_{v}=25$ or $2 Q_{v}=10$, which are not simulated. Apart from the halo, emittances compare rather well and the agreement is satisfactory.

The comparison between Agile and Accsim is even more convincing, taking into account that, due to different algorithms of the two codes, the distribution of the injected beam is only approximately the same: a truncated (at $3 \sigma$ ) Gaussian in Agile and a binomial distribution $(m=3.5)$ in Accsim, both matched to have the same variance and the same finite radii.

\section{REFERENCES}

[1] Leslie F. Greengard, The Rapid Evaluation of Potential Fields in Particle Systems, Cambridge, Mass: MIT Press, 1988.

[2] F.W. Jones, "A Hybrid Fast-Multipole Technique for SpaceCharge Tracking With Halos," Workshop on Space Charge Physics in High Intensity Hadron Rings, Shelter Island NY, May 1998, AIP Conf. Proc. 448, p. 359.

[3] R. Baartman, "Betatron Resonances with Space Charge," ibid, p. 56.

[4] P. Bryant, personal communication and Agile WWW Page, http://nicewww.cern.ch/ bryant.

[5] J.A. Holmes et al., "A Particle-In-Cell Model for SpaceCharge Dynamics in Rings," Proc. European Particle Accelerator Conference, Stockholm, 1998.

[6] Accsim home page, http://www.triumf.ca/compserv/accsim.html. 